\title{
A CHARACTERIZATION OF REFLEXIVITY BY THE LATTICE OF CLOSED SUBSPACES
}

\author{
E. E. FLOYD ${ }^{1}$ AND V. L. KLEE ${ }^{2}$
}

1. Introduction. Smulian [12] proved that a convex subset of a normed linear space fails to be weakly compact if and only if it contains a decreasing sequence of relatively closed nonempty convex sets whose intersection is empty. (His proof is most readily accessible in [8]. Dieudonné [4] has recently proved the theorem in a more general setting.) We establish here a somewhat stronger ${ }^{3}$ result: a bounded closed convex subset of a normed linear space fails to be weakly compact if and only if there is a decreasing sequence of closed linear manifolds ${ }^{4}$ whose intersection is empty and each of which intersects the set. Applied to the unit cell $\{x:\|x\| \leqq 1\}$ of the space, this yields a new characterization of reflexivity. Using this characterization, we obtain our second principal result: a normed linear space is reflexive if and only if the lattice of all its closed linear subspaces is, in the order topology of Birkhoff [2], a Hausdorff space. (The order topology is defined in §3.)

2. A strengthening of Šmulian's theorem. In the work of this section, the notion of nonsupport point plays an important role. If $X$ is a subset of a normed linear space $E$ and $p$ is a point of $X$, then $p$ is a nonsupport point of $X$ provided every hyperplane ${ }^{5}$ which supports $X$ at $p$ actually contains $X$. (That is, whenever $f$ is a continuous linear functional on $E$ such that $\sup _{X} f=f p$, then also $\inf _{X} f=f p$.) We need the following fact.

(2.1) In a normed linear space $E$, each separable complete convex set $C$ has at least one nonsupport point.

Proof. (An equivalent result is stated in [7], using a slightly different definition of nonsupport point, but the proof given there is

Presented to the Society, December 30,1953; received by the editors August 14 1953 and, in revised form, January 22, 1954.

1 Work on this paper supported by National Science Foundation grant NSFG358.

2 Work on this paper sponsored in part by the Office of Ordnance Research, U. S. Army, under Contract DA-04-200-ORD-292.

${ }^{3}$ Although this does not formally include Šmulian's result when the set is unbounded or not closed, these cases are easily handled directly. (See the proof of (2.5).)

- A linear manifold is a translate of a linear subspace.

5 A hyperplane is a closed set which is maximal with respect to being a linear manifold properly contained in the space, or, equivalently, a set of the form $\{x: f x=c\}$, where $c$ is a real constant and $f$ is a continuous linear functional not identically zero. 
valid only when $C$ is compact. The proof given here is due to $\mathrm{E}$. A. Michael.) We assume without loss of generality that $C$ includes the neutral element $\phi$, and let $x_{\alpha}$ be a sequence of points dense in $C$, with $x_{0}=\phi$. For each $n$, let $y_{n}=x_{n}$ if $\left\|x_{n}\right\| \leqq 1$ and $y_{n}=\left\|x_{n}\right\| \|^{-1} x_{n}$ if $\left\|x_{n}\right\|>1$. Let $p=\sum_{0}^{\infty} 2^{-i} y_{i}$. Then $p \in C$, for the series is convergent and each partial sum represents a point of $C$. Now if $f$ is an arbitrary continuous linear functional on $E$ such that $f p \geqq \sup _{c} f$, then $f p$ $=\sum_{0}^{\infty} 2^{-i} f y_{i}$, where always $f y_{n} \leqq f p$. This implies that always $f y_{n}=f p$, whence $f p=f y_{0}=0$ and always $f x_{n}=0$. Since $x_{\alpha}$ is dense in $C, f C=\{0\}$ and the proof is complete.

We need also

(2.2) Suppose $C$ is a convex subset of a normed linear space $E, p$ is a nonsupport point of $\mathrm{cl} C$ (the closure of $C$ ) and $H$ is a hyperplane in $E$ with $p \in H$. Then $p$ is a nonsupport point of $\mathrm{cl}(C \cap H)$.

Proof. We may assume without loss of generality that $p=\phi$ and $C$ is not contained in any hyperplane in $E$. For $X \subset E$, let cone $X=\{t x: t$ $\geqq 0, x \in X\}$. Then cone $C$ is a convex cone in $E$ with $\phi$ as vertex. Now if cone $C$ is not dense in $E$, it follows from [7, p. 457] that cone $C$ is supported at $\phi$ by a hyperplane, contradicting the fact that $\phi$ is a nonsupport point of $C$. Thus cone $C$ is dense in $E$. Now let $q \in E-H$ and consider an arbitrary nonempty convex subset $U$ of $H$ which is open relative to $H$. Let $\left.U^{+}=U+\right] 0,1\left[q\right.$ and $\left.U^{-}=U+\right]-1,0[q$. Then $U^{+}$and $U^{-}$are open subsets of $E$ so must be intersected by cone $C$, whence it follows that cone $C$ intersects $U$. Thus (cone $C$ ) $\cap H$ is dense in $H$, and since this is the same as cone $(C \cap H)$, it follows that $p(=\phi)$ is a nonsupport point of $\mathrm{cl}(\mathrm{C} \cap H)$.

The basic result of this section is

(2.3) Theorem. Suppose $C$ is a bounded closed convex subset of the separable normed linear space $E$, and that $C$ is not weakly compact. Then there is in $E$ a sequence $H_{\alpha}$ of hyperplanes such that with $L_{n}$ $=\bigcap_{1}^{n} H_{i}$, each $L_{n}$ intersects $C$ but $\bigcap_{1}^{\infty} H_{i}$ is empty.

Proof. Let $E^{\prime}$ denote the completion of $E$ and (for $X \subset E$ ) $X^{\prime}$ the closure of $X$ in $E^{\prime}$. Since $C^{\prime}$ is separable, complete, and convex, it follows from (2.1) that the theorem can be proved by proving it for each of the following cases: (a) $C$ has a nonsupport point; (b) $C^{\prime}$ has a nonsupport point which is not in $C$.

Case (a). We may assume that $\phi$ is a nonsupport point of $C$. Since $C / 2$ is not weakly compact, there is a net $(S,>)$ in $C / 2$ which has no weakly convergent subnet. $((S,>)$ is a function whose domain $D_{S}$ is a set directed by the relation $>$ and whose range is contained in $C / 2$. For the terminology and notation of net-convergence, see 
Kelley [6].) By the standard type of argument using Tychonoff's product theorem as a substitute for a "diagonal" process, $(S,>)$ admits a subnet $(T,>)$ such that for each $f \in E^{*},(f T,>)$ converges to a real number $c_{f}$. (Details are given in the next paragraph. This was suggested by E. J. McShane and is simpler than our original argument.)

For each $f \in E^{*}$ let $K_{f}=\left[\inf _{C} f, \sup _{c} f\right]$, and let $P=\prod_{f \in E^{*}} K_{f}$. Then $P$ is compact. For each $u \in D_{S}$, let $\sigma u=f S u \mid f \in E^{*}$. Then $(\sigma,>)$ is a net in $P$ and by compactness of $P$ must admit a convergent subnet $(\tau,>)$. (See [6].) To say that $(\tau,>)$ is a subnet of $(\sigma,>)$ is to say that there is a function $N$ on $D_{\tau}$ to $D_{\sigma}$ such that $\tau=\sigma N$ and such that for each $p \in D_{\sigma}$ there is a $q \in D_{\tau}$ such that $N w \geqq p$ whenever $w \in D_{\tau}$ and $w \geqq q$. Now for each $v \in D_{r}$, let $T v=S N v$. Since $D_{S}=D_{\sigma}$, $(T,>)$ is a subnet of $(S,>)$ and for each $f \in E^{*},(f T,>)$ is the projection of $(\tau,>)$ onto $K_{f}$ and must therefore converge to a number $c_{f} \in K_{f}$.

Now for each $f \in E^{*}$, let $H_{f}=\left\{x: x \in E\right.$ and $\left.f x=c_{f}\right\}$. Then $y$ $\in \bigcap_{f \in E^{*}} H_{f}$ implies that $(T,>)$ is weakly convergent to $y$, so $\bigcap_{f \in E^{*}} H_{f}$ must be empty. Since $E$ is separable it follows by Lindelöf's theorem that there is a countable set of the $H_{f}$ 's whose intersection is empty. To complete the proof (for case (a)) we shall show that for an arbitrary finite subset $\left\{f_{1}, \cdots, f_{m}\right\}$ of $E^{*}, \bigcap_{1}^{m} H_{f_{i}}$ intersects $C$.

For each $x \in E$, let $F x=\left(f_{1} x, \cdots, f_{m} x\right) \in E^{m}$. Then $F(C / 2)$ is a convex set and it is easily verified that $\phi$ is a nonsupport point of $F(C / 2)$. In $E^{m}$, this implies that $\phi$ is interior to $F(C / 2)$ and hence that cl $F(C / 2) \subset 2 F(C / 2)=F C$. Thus we need merely show that $\left(c_{f_{1}}, \cdots, c_{f_{m}}\right) \in \mathrm{cl} F(C / 2)$. But this is evident, for $C / 2$ contains the range of $T$ and $\left(f_{n} T,>\right)$ converges to $c_{f_{n}}$ for each $n$. The proof for case (a) is complete.

Case (b). For this case (unlike the first one) it is unnecessary to assume that $C$ is bounded. Let $p$ be a nonsupport point of $C^{\prime}$ with $p \notin C$. Since $E^{\prime}$ is separable, there is a sequence $H_{\alpha}^{\prime}$ of hyperplanes in $E^{\prime}$ such that $\bigcap_{1}^{\infty} H_{i}^{\prime}=\{p\}$. For each $n$, let $L_{n}^{\prime}=\bigcap_{1}^{n} H_{i}^{\prime}, H_{n}=H_{n}^{\prime} \cap E$, and $L_{n}=\bigcap_{1}^{n} H_{i}=L_{n}^{\prime} \cap E$. It follows from (2.2) that always $p$ is a nonsupport point of $\left(L_{n}^{\prime} \cap C\right)^{\prime}$, and hence in particular that $L_{n}^{\prime} \cap C$ is nonempty. But $C \subset E$ and $p \notin E$ (since $C$ is closed in $E$ and $p \in C^{\prime}$ $-C)$ so we have $L_{n} \cap C=L_{n}^{\prime} \cap C \neq \Lambda$ and $\cap_{1}^{\infty} H_{i}=\{p\} \cap E=\Lambda$, completing the proof.

In a nonseparable normed linear space, there may not exist any sequence of hyperplanes of the sort described in (2.3), but we have still the following result, which was stated in the introduction. 
space fails to be weakly compact if and only if there is a decreasing sequence of closed linear manifolds whose intersection is empty and each of which intersects $C$.

Proof. Since each closed linear manifold is weakly closed, the "if" part is obvious. On the other hand, if $C$ fails to be weakly compact, then from the equivalence of weak compactness with weak sequential compactness (proved in [5]) it follows that some separable closed convex subset of $C$ fails to be weakly compact, whence the desired conclusion follows from (2.3).

Neither the boundedness nor closedness assumption on $C$ can be removed in (2.4). To see this, consider an arbitrary unbounded or nonclosed finite-dimensional convex set. However, for arbitrary convex $C$ we can prove the theorem of Smulian as a corollary of (2.4).

(2.5) Corollary. A convex subset $C$ of a normed linear space $E$ fails to be weakly compact if and only if it contains a decreasing sequence of relatively closed nonempty convex sets whose intersection is emply.

Proof. As before, the "if" part is obvious. Suppose conversely that $C$ fails to be weakly compact and let $C^{\prime}$ be as in the proof of (2.3). We consider three possible cases: (a) $C$ is bounded and closed. Here (2.4) applies at once to yield the desired conclusion. (b) $C \neq C^{\prime}$. Then with $p \in C^{\prime}-C$ and $C_{n}=\left\{x: x \in C\right.$ and $\left.\|x-p\| \leqq n^{-1}\right\}, C_{\alpha}$ is the desired sequence of sets. (c) $C$ is unbounded. From the uniform boundedness principle $[1$, p. 80$]$ it follows that there is an $f \in E^{*}$ such that $\sup _{c} f=\infty$, and with $C_{n}=\{x: x \in C$ and $f x \geqq n\}, C_{\alpha}$ is the desired sequence.

We conclude this section with the characterization of reflexivity which will be a fundamental tool in $\S 3$.

(2.6) Corollary. A normed linear space is nonreflexive if and only if it contains a decreasing sequence of closed linear manifolds whose intersection is empty and each of which intersects its unit cell.

Proof. Since the space is reflexive if and only if its unit cell is weakly compact [5], this result follows at once from (2.4). (Notice that only case (a) of (2.3) is needed here, and that the unit cell obviously has its center as a nonsupport point.)

We remark that (2.4)-(2.6) remains valid if "separable" be inserted after "sequence of" in each case. This is obvious from the proofs given and can also be deduced directly from the statements of the theorems. 
3. The lattice of closed subspaces. For a complete lattice $L$ (with meet and join denoted by $\wedge$ and $\vee$ respectively), G. Birkhoff [2, p. 60] has defined the order topology as follows: A net $(\xi,>)$ in $L$ is order-convergent to the element $x \in L$ provided $\bigvee_{a \in D}\left[\Lambda_{a \leqq b \in D} \xi b\right]$ $=x=\bigwedge_{a \in D}\left[\bigvee_{a \leqq b \in D} \xi b\right]$. A set $X \subset L$ is closed provided $p \in X$ whenever some net in $X$ is order-convergent to $p$. As thus topologized, $L$ is a $T_{1}$-space. Birkhoff $[2$, p. 60$]$ asserted that $L$ must be a Hausdorff space, but it follows from our Theorem (3.3) below (and also from results of Northam [10] and Botts, Floyd, and McShane [3]) that this is incorrect. For additional information on order-convergence and related concepts see $[2 ; 9 ; 11]$.

Now suppose $E$ is a normed linear space, $K$ is the set of all closed convex subsets of $E$ (including the empty set) and $\mathcal{L}$ is the set of all closed linear subspaces of $E$ (not including the empty set, though it makes no difference here). As ordered by set-theoretic inclusion, $K$ is a complete lattice and $\mathcal{L}$ a complete sublattice of $K$. For $A, B \in K$, $A \wedge B=A \cap B$ and $A \vee B$ is the closed convex hull of $A \cup B$ (which, for $A$ and $B$ in $\mathcal{L}$, is identical with the closed linear extension of $A \cup B)$.

For a subset $X$ and an element $p$ of a lattice with order-relation $>$, we write $X \nearrow p$ to indicate that $X$ is directed by $>$ and $\vee X=p$; $X \searrow p$ means that $X$ is directed by $<$ and $\Lambda X=p$.

We shall now prove, in two steps, the second principal theorem stated in the Introduction.

(3.1) Suppose $E$ is a reflexive normed linear space and $K$ is the ordertopologized lattice of all closed convex subsets (including the empty set) of $E$. Then for each pair $A$ and $B$ of distinct members of $K, K$ admits a real-valued continuous monotone function $f$ such that $f A \neq f B$.

Proof. The only property of the order topology needed for this proof is that a real-valued isotone (= "order-preserving") map on $K$ is continuous provided (i) $\mathscr{X} \subset K$ and $\mathscr{X} \nearrow J \in K$ implies $h \mathcal{H} \nearrow h J$ and (ii) $\mathscr{H} \subset K$ and $\mathscr{H} \searrow K \in K$ implies $h \mathcal{X} \backslash h K$. (See [9, p. 24].)

We shall actually define a map $g$ into $[0, \infty]$, but an obvious modification yields the desired map $f$ into $[0,1]$. Suppose $A \nsubseteq B$. Then there is a point $p \in A-B$. For each $C \in K$ let $g C=\inf _{x \in C}\|x-p\|$. Clearly $g A \neq g B,-g$ is isotone, $g \Lambda=\infty$, and $g(K-\{\Lambda\}) \subset[0, \infty[$. It is obvious that $-g$ satisfies (i). Thus to establish continuity of $g$ it suffices to show that if $\mathcal{N} \subset K$ and $\mathcal{N} \searrow K \in K$, then $g \mathcal{N} / g K$. If $\Lambda \in \mathcal{N}$, then $K=\Lambda$ and the conclusion is obvious, so suppose $\Lambda \notin \mathcal{N}$. Since $E$ is reflexive, its unit cell $U$ must be weakly compact. And each closed convex set is weakly closed, so for each $N \in \mathcal{N}$ there is a point $z_{N} \in N$ such that $\left\|z_{N}-p\right\|=g N$. Now if the net $\left(\left\|z_{N}\right\| \mid N \in \mathcal{N}, \subset\right)$ con- 
verges to $\infty$, then $K=\Lambda$ and $g N \nearrow \infty=g K$. If the net $\left(\left\|z_{N}\right\| \mid N \in \mathcal{N}, \subset\right)$ does not converge to $\infty$, then $\left\{\left\|z_{N}\right\|: N \in \mathcal{N}\right\}$ must be bounded, and by weak compactness of $U$ the net $\left(z_{N} \mid N \in \mathcal{N}, C\right)$ must admit a subnet $(\xi,<)$ which converges weakly to a point $q \in K$. Since $U$ is weakly closed, $q$ must be included in every set $p+t U$ which contains some subnet of $(\xi,<)$, and hence $\|q-p\|<\lim$ inf $\|p-\xi\|$

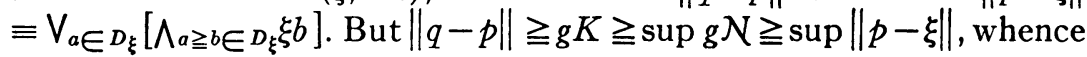
$g K=\sup g \mathcal{N}$ and the proof of (3.1) is complete.

(3.1) shows that when $E$ is reflexive the lattice $K$ is normal in the sense of McShane [9, p. 26], and in particular is a Hausdorff space in the order topology.

(3.2) Suppose $E$ is a nonreflexive normed linear space and $\mathcal{L}$ is the order-topologized lattice of all closed linear subspaces of $E$. Suppose $\mathfrak{W}$ is an open subset of $\mathcal{L}$ and $\mathscr{W} \exists \subset B \in \mathcal{L}$. Then $B \in \mathrm{cl} \mathfrak{W}$.

Proof. We need only the following properties of $\mathscr{W}$ and $\mathrm{cl} \mathscr{W}$ : (i) if $\mathscr{X} \subset \mathcal{L}, L \in \mathscr{W}$, and either $\mathfrak{x} \nearrow L$ or $\mathscr{X} \searrow L$, then $\mathscr{H}$ intersects $\mathscr{W}$; (ii) if $\mathcal{N} \subset \mathrm{cl} \mathscr{W}$ and either $\mathcal{N} \nearrow J$ or $\mathcal{N} \searrow J$, then $J \in \mathrm{cl} \mathscr{W}$.

Let $A$ be the set of all finite-dimensional subspaces of $A$. Then $A \nearrow A$ and hence there exists $C \in W \cap A$. Let $B$ be the set of all finitedimensional subspaces of $B$ which contain $C$. Then $B \nearrow B$ and hence $\mathscr{B} \subset \mathrm{cl} \mathscr{W}$ implies $B \in \mathrm{cl} \mathscr{W}$. Thus to prove (3.2) it suffices to deal only with the case in which $A$ and $B$ are both finite-dimensional. We henceforth restrict our attention to this case.

With $U$ denoting the unit cell of $E$, it follows from (2.6) that there is a decreasing sequence $L_{\alpha}$ of separable closed linear manifolds in $E$ whose intersection is empty and each of which intersects $U$. Since $B$ is finite-dimensional, $L_{\alpha} \oplus B \searrow B$, and thus to complete the proof of (3.2) it suffices to show that cl $\mathscr{W}$ includes each set $M_{n}=L_{n} \oplus B$. (For subsets $X$ and $Y$ of $E, X \oplus Y$ will denote the closed linear extension of $X \cup Y$.) Let $U_{n}=M_{n} \cap U$ and let $S_{0}=A$. Let $x_{\alpha}$ be a sequence of points dense in $M_{n}$, with $x_{0}=\phi$. We wish to define an increasing sequence $S_{\alpha}$ of finite-dimensional subspaces of $M_{n}$ such that always $S_{i} \in \mathcal{W}$ and $S_{i}$ intersects $x_{i}+U_{n} . S_{0}$ has already been chosen. And having chosen $S_{k-1}$, we choose $S_{k}$ as follows: Since $\left(x_{k}+L_{\alpha}\right)$ $\oplus S_{k-1} \searrow S_{k-1}$, there is a $j>n$ such that $\left(x_{k}+L_{j}\right) \oplus S_{k-1}=T \in \mathscr{W}$. There is a point $p=x_{k}+x_{j} \in\left(x_{k}+U_{n}\right) \cap T$, and if $\mathcal{T}$ is the set of all finitedimensional subspaces which contain $\{p\} \oplus S_{k-1}$ and are contained in $T$, then $\mathcal{T} \nearrow T$. Thus some member of $\mathcal{T}$ is in $\mathscr{W}$ and can be chosen as $S_{k}$. Proceeding by mathematical induction, the sequence $S_{\alpha}$ is obtained as described. Now $\bigcup_{1}^{\infty} S_{i}$ intersects $x_{i}+U_{n}$ for each $i$, so $S_{\alpha} \nearrow M_{n}$ and $M_{n} \in \mathrm{cl} \mathscr{W}$, completing the proof of (3.2). 
Combining (3.1) and (3.2) we have the following theorem, which was announced in the Introduction:

(3.3) Theorem. Suppose $E$ is a normed linear space and $\mathcal{L}$ is the order-topologized lattice of all closed linear subspaces of $E$. Then $E$ is reflexive if and only if $\mathcal{L}$ is a Hausdorff space.

\section{BIBLIOGRAPHY}

1. Stefan Banach, Théorie des operations linéaires, Warsaw, 1932.

2. Garrett Birkhoff, Lattice theory, Amer. Math. Soc. Colloquium Publications, vol. $25,1948$.

3. T. A. Botts, E. E. Floyd, and E. J. McShane, Lattices with pathological order topologies.

4. J. Dieudonné, Sur un théorème de Šmulian, Archiv der Math. vol. 3 (1953) pp. 436-440.

5. W. F. Eberlein, Weak compactness in Banach spaces. I. Proc. Nat. Acad. Sci. U.S.A. vol. 33 (1947) pp. 51-53.

6. J. L. Kelley, Convergence in topology, Duke Math. J. vol. 17 (1950) pp. 277-283.

7. V. L. Klee, Jr., Convex sets in linear spaces, Duke Math. J. vol. 18 (1951) pp. $443-466$.

8. - Convex sets in linear spaces. II., ibid. pp. 875-883.

9. Edward J. McShane, Order-preserving maps and integration processes, Annals of Mathematics Studies, no. 31, 1953.

10. E. S. Northam, Topology in lattices, Bull. Amer. Math. Soc. Abstract 59-4-429.

11. Basil C. Rennie, The theory of lattices, Cambridge, 1951.

12. V. Smulian, On the principle of inclusion in the space of the type (B), Mat. Sbornik (N.S.) vol. 5 (47) (1939) pp. 317-328.

UNIVERSITY OF VIRGINIA AND

UNIVERSITY OF WASHINGTON 